Slavica

bruxellensia

\section{Slavica bruxellensia}

Revue polyphonique de littérature, culture et histoire

slaves

5 | 2010

Après 1989

\title{
La littérature serbe avant et après la chute du mur de Berlin
}

\section{Milivoj Srebro}

\section{OpenEdition \\ Journals}

Édition électronique

URL : http://journals.openedition.org/slavica/295

DOI : $10.4000 /$ slavica.295

ISSN : 2034-6395

\section{Éditeur}

Université libre de Bruxelles - ULB

\section{Édition imprimée}

Pagination : 7-20

ISSN : 2031-7654

Référence électronique

Milivoj Srebro, "La littérature serbe avant et après la chute du mur de Berlin », Slavica bruxellensia [En ligne], 5 | 2010, mis en ligne le 15 février 2010, consulté le 03 mai 2019. URL : http:// journals.openedition.org/slavica/295; DOI : 10.4000/slavica.295

Ce document a été généré automatiquement le 3 mai 2019.

\section{(c) $($ i) $(9)$}

Les contenus de Slavica bruxellensia sont mis à disposition selon les termes de la Licence Creative Commons Attribution - Pas d'Utilisation Commerciale - Pas de Modification 3.0 France. 


\title{
La littérature serbe avant et après la chute du mur de Berlin
}

\author{
Milivoj Srebro
}

\section{Introduction}

1 La chute du Mur de Berlin, qui a sonné le glas du communisme en Europe n'a pas eu le même impact sur la vie intellectuelle et littéraire dans tous les anciens pays communistes. Si cet important événement historique a effectivement ouvert une nouvelle ère dans l'espace culturel de l'ex-bloc soviétique - une ère où les écrivains pouvaient enfin espérer jouir pleinement de la liberté d'expression -, il s'est inscrit dans un contexte socioculturel différent en ex-Yougoslavie, pays qui se trouvait alors déjà au bout du gouffre. Certes, la fin du communisme dans ce pays a également éveillé l'espoir, du moins dans les milieux intellectuels de l'obédience démocratique, d'une démocratisation possible de la sphère culturelle et de la société yougoslaves affectées par une crise profonde; un espoir aussi d'une libération définitive des contraintes idéologiques et de la pensée unique que le régime titiste puis celui de ses successeurs avaient imposées aux écrivains et aux artistes. Mais cet espoir fut malheureusement de courte durée : la disparition du communisme a permis aux écrivains, il est vrai, de se débarrasser à jamais de l'héritage du titisme mais, au même temps, elle fut le prélude à une nouvelle époque particulièrement agitée, dominée par les événements tragiques engendrés par la guerre civile. Ainsi, au lieu de profiter, à l'instar de leurs collègues de l'Est, des avantages propres à une société démocratique respectant le droit à la libre expression, les écrivains de l'ex-Yougoslavie durent faire face à une situation inédite, extrême, peu favorable à une activité intellectuelle sereine.

2 Aussi, pourrait-on observer une différence notable dans l'évolution, d'une part des littératures des pays de l'Est et, d'autre part, des littératures de l'ex-Yougoslavie durant la période précédant l'écroulement du communisme. Plus précisément, les écrivains yougoslaves, contrairement à leurs confrères de l'ex-bloc soviétique, ne furent pas contraints d'attendre la chute du Mur de Berlin pour jouir d'une liberté accrue qui - faut- 
il le préciser ? - ne leur fut pas accordée mais qu'ils avaient conquise eux-mêmes. Sur le mécanisme d'une censure sournoise exercée par le régime titiste et les successeurs de Tito, mais aussi sur la manière dont on pouvait le déjouer grâce à l'audace personnelle comportant, bien entendu, un certain risque, témoigne le livre Écrire sous surveillance ${ }^{11} \mathrm{de}$ Milovan Danojlić, poète et romancier serbe depuis longtemps installé en France :

Chez nous, rien ou presque rien n'est explicitement interdit. La censure dans le vrai sens du terme, n'existe pas. Les manuscrits ne sont pas soumis à l'examen d'un représentant de l'État et ne reçoivent pas imprimatur d'en haut. Le contrôle est effectué par les comités de lecture, les directeurs de collection, les rédacteurs en chef et autres lecteurs, correcteurs ou délateurs occasionnels, mais le frein principal se trouve dans la tête de l'auteur: chacun détermine seul ce qui est indésirable et socialement inacceptable. (...) À chacun donc de décider jusqu'où il peut aller trop loin. Les courageux élargiront le champ du possible alors que les timorés étendront tout naturellement le domaine de l'interdit. (...) Tout ce que nos persécuteurs entreprennent est, en définitive, vain et sans lendemain. Il suffit d'endurer certains moments pénibles. (pp. 11-13).

C'est justement grâce à ce courage personnel et au prix «d'endurer certains moments pénibles » que les écrivains yougoslaves réussirent à sauvegarder une certaine liberté de pensée avant de se lancer avec plus de vigueur dans sa conquête. Précisément, la première grande occasion pour mettre ouvertement au défi le régime, et même pour engager un véritable bras de fer avec les autorités de leur pays, s'est présentée à eux en 1980, l'année de la mort de Josip Broz Tito, fondateur et président à vie de la Yougoslavie socialiste. D'ailleurs, c'est cet événement, la disparition de Tito, qui représente également une date charnière dans l'évolution de la littérature serbe contemporaine, événement sans lequel, à vrai dire, il n'est pas possible de saisir toutes les particularités de la vie littéraire postérieure en Serbie.

La disparition de Tito, après un long règne de trente cinq ans, marque en effet la fin d'une époque et le début d'une autre, beaucoup plus agitée, caractérisée par des crises économiques et politiques successives, qui finira de façon dramatique par la guerre civile et l'effondrement du pays en 1991. Les intellectuels et plus particulièrement les écrivains serbes furent parmi les premiers en ex-Yougoslavie à sentir que la disparition de Tito causerait tôt ou tard la fin d'un régime autoritaire longtemps dissimulé par la personnalité charismatique du Président. Conscients que l'État commun des Slaves du Sud ne pouvait plus fonctionner comme auparavant - c'est-à-dire par le recours à une idéologie autoritaire reposant sur le mythe de la "fraternité et unité » (bratstvo-jedinstvo), sur l'histoire trafiquée et sur des méthodes sournoises de répression - ils ne restèrent pas longtemps les bras croisés. Las du jeu du chat et de la souris qu'ils avaient été obligés de mener avec les autorités, ils décidèrent cette fois de formuler un certain nombre de revendications, exprimées parfois de façon directe, parfois sous forme littéraire, tout en sachant qu'ils risquaient fort bien de s'attirer les foudres des "gardiens du temple », les successeurs de Tito. Sans se douter d'où venait le danger, le régime - qui avait déjà lancé un nouveau slogan comme message clair à ceux qui rêvaient de démocratisation de la société : « Après Tito, encore Tito $\|^{2}-$, n'hésita pas effectivement à répondre par la force à ces revendications qu'il considérait comme des «provocations». En les qualifiant "d'actes contre l'État et le peuple», il alla jusqu'à l'interdiction de livres et même à l'arrestation de leurs auteurs. 
5 Longtemps contraints de se tenir à l'écart de tout ce qui avait trait au mythe et à l'idéologie communistes, longtemps privés de la possibilité d'aborder ou, tout simplement, d'évoquer certains événements de l'histoire récente que le régime avait voulu gommer de la mémoire collective, les écrivains lancèrent d'abord un défi au pouvoir en s'attaquant aux derniers thèmes-tabous. Parmi eux, trois avaient été frappés, jusqu'à la mort de Tito, d'absolus interdits: la personnalité charismatique et le rôle historique du "père de la nation", le génocide commis par les oustachis contre la population serbe durant la Deuxième Guerre mondiale, et la répression massive - purges, arrestations, tortures - organisée par le régime contre ceux suspectés d'avoir conservé leur allégeance à Staline, après la rupture entre celui-ci et Tito en 1948.

Le premier de ces thèmes-tabous était sans doute de loin le plus délicat à aborder. Et le poète Gojko Djogo - qui a eu le courage de s'en prendre à Tito, à travers une allégorie mordante développée dans son livre Le Temps de laine (1981) -, paya cher son audace. Accusé d'outrage au culte de la personnalité, le poète fut condamné à deux ans de prison ferme et son livre, bien entendu, mis à l'index. D'autres écrivains, traitant d'autres thèmes-tabous, s'en tirèrent tout de même mieux, bien qu'ils aient dû, eux aussi, subir les calomnies et les critiques acharnées des idéologues communistes. Il s'agit notamment de Jovan Radulović, Vuk Drašković et Antonije Isaković. Les deux premiers - dans leurs œuvres respectives, le drame Golubnjača (1980) et le roman Le Couteau (1982) - firent éclater l'une des vérités soigneusement cachées par les historiens officiels, en mettant à nu l'idéologie raciste des oustachis et leurs effroyables méthodes d'extermination appliquées sur les Serbes. Quant au troisième, Isaković, il a fait découvrir aux lecteurs, dans son roman L'instant II (1982), le monde particulièrement cruel des prisonniers politiques du tristement célèbre camp de Goli otok (l'île Nue, au large de la côte adriatique) - « le goulag yougoslave $»^{3}$.

7 Bien qu'ils furent fortement critiqués, surtout Golubnjača et Le Couteau*, ces livres «hérétiques" marquèrent des points importants dans la partie de bras de fer qui se jouait entre le régime et l'intelligentsia serbe ${ }^{4}$. D'autre part, ils avaient ouvert la voie à d'autres livres de fiction, toujours plus nombreux, qui eux aussi s'attaquaient à des thèmes proscrits. Ainsi, en 1982, Slobodan Selenić fait paraître le roman Lettre-Tête où il évoque le monde concentrationnaire de l'île Nue. L'année suivante, en 1983, paraît la comédie de Dušan Kovačević, l'Espion balkanique, où l'auteur démasque la psychologie claustrophobe, l'un des produits typiques de l'idéologie titiste. En 1984, Danko Popović publie son roman Le Livre de Miloutine* où il met en lumière les souffrances d'un paysan serbe, infligées par le pouvoir communiste. Finalement, à partir de 1985, Dobrica Ćosić commence à éditer les volumes de sa trilogie Le Temps du mal*, un véritable réquisitoire contre cette idéologie néfaste qui, tout en promettant un avenir radieux, a causé tant d'illusions perdues, de souffrances et de morts. Les livres cités jouèrent donc un rôle prépondérant dans le processus de "détitisation" de la sphère culturelle serbe et yougoslave. Mais, bien entendu, ils n'étaient pas les seuls à être publiés durant cette époque. En fait, ces livres font partie d'un courant littéraire qui n'a cessé de s'affirmer au fil des années, un courant critique et contestataire vis-à-vis de «l'histoire » et de «la vérité » officielles, dominé par un fort accent politique, allant parfois à l'encontre des qualités esthétiques. D'autre part, il faut souligner que parallèlement à ce courant très politisé, et souvent mêlé à lui, évolue un autre courant littéraire, plus hétérogène et plus diversifié quant aux choix des thèmes et des procédés littéraires. Il englobe des écrivains d'orientations poétiques diverses mais qui ont en commun de rejeter l'engagement 
politique direct par le biais de la littérature. Sans éluder les sujets en prise avec la réalité, ceux-ci n'hésitent pas non plus à aborder les questions épineuses de l'histoire et de l'actualité, mais d'une façon plus propre à la littérature qui leur permet, en même temps, de satisfaire leurs exigences d'ordre esthétique et de s'aventurer dans des recherches de forme, de style ou de langage.

Parmi ces écrivains, une place à part revient à Milorad Pavić, grand amateur des procédés insolites et des formes littéraires déroutantes, qui dessine, dans ses livres, un univers fantasque afin de démontrer que la réalité, telle que nous l'apercevons, n'est qu'une apparence trompeuse. Esprit ludique, érudit sans pareil et mystificateur hors pair, cet écrivain crée un véritable événement en publiant en 1984 son chef d'œuvre, Le Dictionnaire khazar*, qui deviendra la Bible des "postmodernes" serbes. Conçu comme une encyclopédie romanesque et présenté sous forme d'un roman lexique, ce livre étrange, certainement l'ouvrage le plus original de la littérature serbe, sera traduit par la suite dans le monde entier. L'originalité de cet ouvrage, de ce livre-labyrinthe qui tient de la Tour de Babel, réside d'abord dans sa forme insolite, expression d'un projet littéraire quasiment révolutionnaire dont le but était non seulement de renouveler l'art de la narration mais aussi de changer le mode traditionnel de lecture !Voici les « instructions » que l'auteur adresse au lecteur dans le "mode d'emploi du dictionnaire» afin de lui faciliter la tâche tout en l'incitant à une lecture créative :

Ainsi le lecteur pourra-t-il utiliser cet ouvrage de façon qui lui plaira. Les uns chercheront un mot ou un nom, comme dans un quelconque dictionnaire, d'autres liront ce livre comme n'importe quel livre, du début à la fin, d'un seul trait, afin d'avoir une vision globale de la question khazare et des personnages, objets et événements qui s'y rapportent. On peut feuilleter ce livre de gauche à droite, ou de droite à gauche... Les trois livres de ce dictionnaire - le jeune, le rouge et le vert seront lus dans l'ordre décidé par le lecteur : il peut commencer par exemple, par celui sur lequel le dictionnaire s'ouvrira... Le Dictionnaire khazar peut se lire également en diagonale afin d'obtenir une coupe à travers les trois livres islamique, chrétien et hébraïque... Ainsi chaque lecteur créera son propre livre comme dans une partie de domino ou de cartes, recevant de ce dictionnaire, comme d'un miroir, autant qu'il y investira, car - c'est écrit dans ce lexique - on ne peut recevoir de la vérité plus qu'on n'y a mis ${ }^{5}$. (pp. 21-22)

9 A côté de Pavić, un certain nombre d'écrivains déjà confirmés, ont montré, durant les années 1980, qu'ils avaient également atteint une pleine maturité, tels que Danojlić, Selenić, Danilo Kiš, Aleksandar Tišma, Borislav Pekić, Filip David, etc. Quant aux poètes, comme, par exemple, Matija Bećković, Ljubomir Simović, Brana Petrović, Milan Komnenić, Slobodan Rakitić, Rajko Petrov Nogo ou Novica Tadić, on observe également chez eux un changement important. De plus en plus inspirés par la réalité, leurs poèmes expriment aussi, par les moyens propres à la poésie, l'atmosphère de crise qui domine l'époque d'après Tito ${ }^{6}$.

10 Pour compléter ce rapide tour d'horizon de la littérature serbe des années 1980, il est indispensable d'évoquer également un troisième courant littéraire conçu sur une idée différente de la littérature. Apparu dans le milieu des jeunes prosateurs - tels que David Albahari, Radoslav Petković, Svetislav Basara, Dragan Velikić, Miroslav Toholj, Nemanja Mitrović, Sreten Ugričić, Mileta Prodanović, Vladimir Pištalo, pour ne citer qu'eux - ce courant dit « postmoderne » ne fut pas non plus homogène ${ }^{7}$. Cette réserve faite, on peut toutefois distinguer quelques points communs chez ces prosateurs de talent inégal. D'abord, un constat d'ordre général : tous partagent le même sentiment, celui de vivre dans un temps d'après. Le monde des fausses certitudes dans lequel ils sont nés commence, 
après la disparition de Tito en 1980, à s'écrouler sous leurs yeux, ce qui ne pouvait qu'accentuer leurs doutes tant à l'égard de la politique et de l'idéologie que de tous les systèmes de pensée fondés sur la raison totalisante. Leurs doutes s'accroîtront encore, bien sûr, $\mathrm{au} \mathrm{vu}$ des événements tragiques ultérieurs. Hostiles à toute forme d'engagement, ces écrivains ont cherché un refuge dans la littérature, mais celle-ci ne pouvait pas non plus leur donner pleine et entière satisfaction: elle leur paraissait usée, renforçait d'autant plus leur sentiment que tout était déjà dit, écrit, et qu'ils sont arrivés après. Pourtant, bien qu'ils crièrent à qui voulait l'entendre qu'écrire n'a plus de sens puisque «à quoi bon répéter ", les " postmodernes » sont parvenus à attirer l'attention sur la nécessité urgente de reconsidérer la logique et le sens de la littérature. Ce faisant, ils ont également proposé de nouveaux modes d'expression et donné une nouvelle dynamique à la littérature serbe durant les années $1980^{\circ}$.

11 Si les «postmodernes» rejetaient toute forme d'engagement politique, la plupart des autres écrivains serbes, en revanche, ont vu dans la disparition de Tito, comme nous l'avons déjà fait remarquer, l'occasion rêvée pour libérer définitivement la littérature des chaînes idéologiques. Après la chute du communisme, en 1989, les langues naturellement se délièrent davantage, ce qui ne servit pas nécessairement "la cause littéraire». D'ailleurs, les tentatives pour éclairer les derniers «trous noirs » de l'histoire nationale récente ne pouvaient s'effectuer bien longtemps dans la sérénité. À peine s'est-elle arrachée aux griffes communistes que la littérature serbe doit à nouveau affronter un contexte socio-historique des plus défavorables. Précisément, au cours des années 1990, «les années noires» de l'histoire serbe et yougoslave, les écrivains sont contraints d'affronter un flot d'événements bouleversants, une véritable spirale infernale qui commence avec la désagrégation du pays et le déclenchement de la guerre civile en 1991, et se termine dans une catastrophe sans précédant, sous les bombes de l'OTAN en 1999. Durant cette période, la Serbie est coupée du monde, mise dans une sorte de quarantaine par un blocus imposé de l'extérieur, ce qui ne pouvait que renforcer le sentiment de vivre dans un "pays de nulle part» ou encore dans un "pays maudit», pour reprendre les titres respectifs des livres de Svetlana Velmar-Janković et Basara. Bref, la littérature se trouvait placée dans un environnement où l'agitation le disputait à la confusion et qui n'était guère propice à une écriture sereine.

12 Déjà avec la chute du communisme, le climat commence à s'échauffer sérieusement sur la scène littéraire serbe. C'est à ce moment-là que la plupart des écrivains prennent part activement aux débats publics dont les sujets dominants sont le réveil national et l'instauration de la démocratie. Sans pouvoir toujours concilier ces deux buts imposés, certains d'entre eux radicalisent leur position et tombent dans les pièges du nationalisme, phénomène qui n'a pas non plus épargné des intellectuels d'autres ex-républiques yougoslaves. En revanche, d'autres, plus vigilants, réussissent à résister à ce virus dangereux mais, sans pouvoir se mettre d'accord quant à la voie à choisir dans leurs relations avec le régime de Slobodan Milošević, ils gaspillent une bonne partie de leur énergie dans des polémiques sans issue et des querelles byzantines.

13 Avec l'arrivée de la guerre civile et de ses cortèges de morts, les écrivains eux aussi se retrouvent dans une situation extrêmement délicate. Obligés d'agir en urgence devant une catastrophe imminente, ils sont contraints de se définir sans équivoque par rapport à la nouvelle situation et de faire un choix souvent irréversible et radical. Ainsi, certains d'entre eux choisissent de s'engager activement dans la politique : tels Ćosić, Drašković, 
Rakitić, Isaković. D'autres, plus nombreux, tentent de faire passer leurs idées par d'autres moyens, le plus souvent à travers les médias choisis selon leur préférence politique : ceux contrôlés par le gouvernement ou ceux fondés par l'opposition. Quelques autres écrivains, enfin, optent pour une troisième voie: décidés à montrer publiquement leur pacifisme et leur opposition au régime belgradois - comme, par exemple, Mirko Kovač, Vidosav Stevanović et Bora Ćosić - ou dégoûtés par la politique, par la désagrégation du pays et par les horreurs de la guerre, comme Tišma ou Albahari, ils quittent leur pays pour s'installer provisoirement ou définitivement ailleurs. Il est évident que, dans une telle situation, leurs prises de positions n'ont pas pu se faire en douceur. Les plumes sont devenues déjà trop grinçantes et les mots trop passionnés pour qu'en sorte un débat constructif. De plus, avec le départ significatif de certains écrivains à l'étranger, les querelles internes ont franchi, elles aussi, les frontières, creusant davantage le fossé entre ceux qui étaient restés et ceux qui étaient partis. La guerre des armes a ainsi fini par provoquer une sorte de "guerre de plumes " qui a eu son écho même dans les médias occidentaux.

Dans ce contexte surchauffé, la littérature serbe n'a pas pu non plus s'en sortir indemne : elle porte évidemment quelques séquelles de cette violente " guerre de plumes » ainsi que des traces de cette profonde crise de la société serbe. Le roman et la nouvelle de cette époque sont - faut-il s'en étonner? - teintés de pessimisme et parfois imprégnés d'un fort sentiment de fatalité et de désespoir; leurs héros sont le plus souvent des individus dépourvus de repères, voués à l'échec, des perdants sans illusions qui observent leur monde grotesque, absurde, avec cynisme et humour noir. Mais, très paradoxalement, malgré la crise générale, y compris celle qui frappe le domaine de l'édition, la littérature des années 1990 démontre une vitalité étonnante qui se reflète et dans le nombre conséquent de livres publiés et dans leur diversité thématique et stylistique. Cette diversité provient, par ailleurs, du croisement de différentes poétiques et de la coexistence de plusieurs générations d'écrivains. Ainsi, par exemple, les nouvelles œuvres des « postmodernes » et de leurs prédécesseurs, les représentants de diverses tendances stylistiques, côtoient des livres de jeunes prosateurs qui pratiquent aussi bien le récit néoréaliste que le récit métanarratif ou fantastique.

Ceci dit, force est de constater tout de même que la littérature serbe a également payé son tribut à l'esprit de l'époque. C'est surtout perceptible dans les deux types d'ouvrage privilégiés par les éditeurs qui étaient proches du régime: celui de l'engagement politique qui, souvent basé sur un schéma manichéen, soutenait "la cause nationale »; celui dit communément "récit de guerre", qui se contentait de susciter de fortes émotions chez le lecteur en jouant sur la fibre patriotique. Heureusement, ces types stéréotypés d'écriture regorgeant de clichés et de lieux communs ne sont pas parvenus à " infecter » les écrivains de talent. Sans fuir la réalité, ceux-ci n'ont pas hésité à s'attaquer aux problèmes épineux de l'actualité, mais avec les moyens propres à la littérature, sans céder aux appels du "devoir patriotique », sans sacrifier la "cause littéraire » au profit de la « cause nationale ». Pour étayer ce constat, il suffit de passer en revue les écrivains et les œuvres qui ont fortement marqué la production littéraire en Serbie durant les années 1990.

Comme nous l'avons fait observer, la chute du communisme a largement ouvert la porte à un examen critique de l'époque titiste et de ses travers. Libérés définitivement des contraintes idéologiques mais aussi de ce qu'impose l'autocensure, de nombreux écrivains 
ont en effet profité de l'occasion pour « ouvrir leur cœur » et dire leur vérité, interdite jusqu'alors. Ainsi, Dragoslav Mihailović qui s'est déjà attaqué auparavant - certes d'une façon détournée, en filigrane - au thème-tabou du "goulag yougoslave ", revient sur ce sujet dans plusieurs de ses livres, notamment dans le recueil La chasse aux punaises (1993) et dans les trois volumes de prose documentaire intitulée simplement L'île nue (1990-1995). Fin connaisseur de « l'âme populaire » qui s'est forgé la réputation de porteparole des déshérités et des humiliés, cet humaniste sensible aux souffrances humaines, en particulier à celles infligées par un régime brutale et hypocrite, se fera également, dans les livres cités, l'avocat compatissant des victimes de la répression communiste en décrivant avec force, à l'instar d'un Soljenitsyne, les mécanismes de l'horrible « système de rééducation » titiste.

Dans son roman Dans le noir* (1990) Velmar-Janković a choisi une approche quelque peu différente de l'époque titiste. Présenté sous la forme de la confession d'une vieille dame issue de l'élite intellectuelle de Belgrade de l'avant-guerre, ce roman a jeté une nouvelle lumière sur le drame de la bourgeoisie belgradoise écartée, bannie ou purement et simplement éliminée après l'instauration du communisme en Yougoslavie. Femme d'un " traître à la patrie ", contrainte à une sorte d'exil intérieur, la narratrice nous livre un témoignage bouleversant sur l'époque du "cruautisme ", la brutalité des vainqueurs et les mécanismes sournois d'un régime totalitaire, un témoignage qui est à la fois une réflexion lucide et nuancée sur le mal engendré par l'histoire. Plus tard, dans le roman Le Pays de Nulle part* (2000), qui pourrait être lu comme la suite du précédent, VelmarJanković revient sur les traumatismes de l'époque titiste. Mais ce livre évoque aussi « les années noires » de la fin du $\mathrm{XX}^{\mathrm{e}}$ siècle et surtout la guerre que l'OTAN a menée contre la Serbie en 1999. Partagée entre la révolte et le désespoir, la romancière y décrit sans complaisance la réalité cauchemardesque d'une guerre tant humiliante que meurtrière entreprise par les grandes puissances pour châtier - comme dans un « jeu vidéo infernal » - un pays tenu pour «le siège du mal ». Voici, comme illustration, un bref extrait de ce roman :

J'ai trouvé les réponses que je chercherais et aussi, me semble-t-il, le droit de me demander si nous, les Serbes, n'allons pas connaître en cette fin de $\mathrm{XX}^{\mathrm{e}}$ siècle ce qu'ont enduré les cathares pendant la première moitié du XIII ${ }^{e}$, même si nous ne constituons pas une communauté hautement cultivée. Je ne sombre pas dans le pathétique... Quelle serait la réaction des habitants de Paris ou de Londres en pareil cas? Ou des habitants de New York? Je doute qu'ils puissent comprendre que le chaos où il leur faut se débattre résulte de la responsabilité qu'on leur fait endosser pour les fautes commises en matière de politique extérieure par leurs ministres ou chef de gouvernement. C'est ridicule, franchement. On ne peut espérer des exterminateurs qu'ils témoignent de la compréhension à ceux qu'ils réduisent à néant, même les cathares en auraient été incapables, j'en suis certaine, seul le Christ y est parvenu, mais lui était le Fils de Dieu. (pp. 144-145)

Parmi d'autres écrivains traitant le même sujet, citons encore le romancier et le nouvelliste Milisav Savić. Attiré par des questions d'ordre éthique, idéologique et existentiel qui se sont posées au fil de temps face à sa génération, ce prosateur a décidé lui aussi de régler ses comptes avec ses illusions de jeunesse, en se faisant en même temps le juge impitoyable de l'ère titiste. Ainsi dans son roman Pain et peur (1991), Savić fera, tout en s'attaquant sans pitié ni nostalgie à son passé de soixante-huitard, un bilan noir de sa génération - la "génération de Tito ", sacrifiée, abusée, et désabusée.

Après l'éclatement de l'ex-Yougoslavie et le déclenchement de la guerre civile, de nouveaux thèmes, encore plus douloureux et plus traumatisants, font leur apparition 
dans la littérature serbe, notamment dans des œuvres des écrivains qui ont quitté le pays, tels Stevanović et Albahari甲. Irréductible opposant au régime de Milošević, le premier s'installe à Paris en 1993 où il achève sa trilogie romanesque en deux volumes - La neige et les chiens* et Christ et les chiens* (1993) - et publie deux nouveaux romans : La même chose* (1999) et Abel et Lise* (2003). Dans ces trois œuvres inspirées par la récente tragédie yougoslave, par ce "fratricide» insensé où "libérateurs" et "défenseurs" se différencient uniquement par «la puanteur de leur haine», Stevanović dépeint, pour reprendre l'expression d'un critique, «l'enfer humain en l'homme et parmi les hommes ». Écrits dans l'urgence, à chaud, ces romans se présentent à la fois comme un hommage rendu aux innocents, victimes de la folie meurtrière des autres, leurs semblables, et comme une véhémente dénonciation de la guerre et de tous les fanatismes qui engendrent inéluctablement la haine et le mal. Citons, à titre d'exemple, un court passage tiré de La neige et les chiens :

Pas de doute, la guerre a commencé. Est-il possible que ce soit moi qui écrive ces mots, de ma main encore vivante? Ceux qui se disent les libérateurs se sont installés sur les collines et les montagnes autour de la ville, et ils tirent sans arrêt. Ceux qui se disent les défenseurs sont partout autour de nous, parmi nous. Et ils tirent. Il n'y a plus de place pour nous en ville. Il ne nous reste que les caves. (...) Ici, on est tous pareils; ceux qui ne se souviennent pas d'une guerre, ils vont en vivre une autre. Comme si c'était en réalité une même guerre qui s'interrompt puis reprend, et ne dépend pas de nous, mais de la volonté de ceux qui aiment faire la guerre. Quand on oublie la mort, il se trouve toujours quelqu'un pour vous la rappeler... (p. 181)

Quant à Albahari, chef de file de la jeune génération des prosateurs des années 1980 et promoteur fervent des idées postmodernistes venues d'outre-Atlantique, un changement notable dans sa prose s'opère en particulier après son départ au Canada. Certes, dans les quatre romans publiés durant les années 1990 - L'Homme de neige* (1995), L'Appât* (1996), L'Obscurité $^{*}$ (1997) et Goetz et Meyer* (1998) - on retrouve toujours les traits caractéristiques de sa poétique: la symbiose ludique d'éléments autobiographiques et fictionnels, les commentaires métanarratifs et le doute permanent à l'égard du langage et de la narration. Mais le champ thématique de ces livres est sensiblement différent : dans l'univers clos et intimiste de ses nouvelles, a fait irruption la Grande Histoire, la bouleversante histoire des Balkans. D'ailleurs, déjà dans le commentaire de L'Homme de neige, rédigé dans une paisible ville du Canada, bien loin de l'histoire balkanique en pleine ébullition, l'auteur a fait comprendre qu'il ne pouvait plus ignorer l'histoire ni la fuir en constatant non sans résignation : " elle est le maître de mon destin ainsi que de celui de nous tous ». Sans se laisser, pour autant, engloutir par l'histoire et tout en essayant, avec les moyens propres à sa poétique postmoderne, de maitriser son «intrusion » dans l'univers fictionnel de sa prose, Albahari revient donc dans ses romans et sur le passé traumatique et sur la réalité douloureuse de son pays. Ainsi, par l'intermédiaire d'un narrateur-écrivain, alter ego de l'auteur, il fait apparaître en filigrane le drame de la récente guerre civile et le climat qui l'a précédé. Il introduit aussi le thème de l'exil, expérience toujours éprouvante, même lorsqu'elle est librement choisie.

21 Le thème de l'exil, grand et douloureux sujet de la littérature du XXe siècle, apparaît également chez Velikić, romancier qui s'intéresse en particulier à la situation existentielle et ontologique de l'homme contemporain et à sa recherche illusoire du bonheur et du sens de la vie. Souvent mis en relation étroite avec un autre thème, celui de la quête de l'identité, l'exil est vu dans ses romans, surtout dans le Mur nord* (1995), avant tout comme une expérience traumatisante, produit de la folie de l'Histoire, mais, dans la 
vision romanesque de cet écrivain, il n'y a pas forcément des connotations exclusivement négatives. Pour ses héros, l'exil, imposé ou choisi, peut être, également, le chemin vers la découverte de soi, de sa véritable identité ou, encore, peut prendre la forme d'une fuite ou d'une quête d'un ailleurs où il serait possible de se forger une nouvelle, une autre identité : dans ce dernier cas, l'exil est vécu comme une sorte d'échappatoire, comme une tentative, évidemment illusoire, de changer la vie et éviter le sort de la mouche « recluse dans l'espace vide d'un double vitrage »

L'exil et la récente guerre civile, en particulier celle qui opposait les Serbes et les Croates de la Krajina, sont également des thèmes récurrents dans les romans et les nouvelles de Radulović (La Vie s'est écoulée, 1997; La place idéale, 2000). Viscéralement lié à la Krajina, son pays natal, et fortement attaché à l'identité culturelle de la diaspora serbe dont il est issu, cet écrivain se fera à la fois le chroniqueur et l'interprète du destin historique de cette même diaspora ; destin émouvant et tragique qui prendra un accent biblique durant l'exode collectif en 1995, à la fin de la guerre civile croato-serbe. Afin de mieux saisir les racines du mal qui a façonné ce destin particulier, Radulović entreprend également, dans ses livres, la relecture du passé et plonge dans l'histoire turbulente de la Krajina tout en s'attachant à démontrer que c'est justement cette histoire volcanique, semée de conflits ethniques et religieux, qui a engendré la haine entre les Serbes et les Croates de cette région; une haine souvent irrationnelle, le vrai détonateur, selon lui, de tous les malheurs, ceux d'hier comme ceux d'aujourd'hui.

Les reflets de la guerre civile et la réalité chaotique qu'elle a engendrée apparaissent aussi, évidemment, dans les livres de Basara, le farouche opposant au régime de Milošević et la figure la plus controversée de la littérature serbe contemporaine. Talent hors pair, esprit frondeur et provocateur, cet écrivain - qui s'était déjà forgé auparavant l'image d'un original prenant un plaisir malin à malmener les règles prescrites et à narguer les défenseurs de la « haute » littérature. - n'a cessé, tout au long du règne du régime de Milošević, de s'attaquer à l'ordre établi. Toujours prêt à jouer l'avocat du diable, il prenait surtout pour cible, quitte à choquer ou à provoquer le scandale, les vérités dites sacrées et les autorités intellectuelles incontestées, sans jamais se prendre trop au sérieux. Durant les années 1990, Basara a publié une demi-douzaine de romans, preuve d'une exceptionnelle capacité d'écriture. Parmi eux, citons en particulier De bello civili, version Vitamine $C^{*}(1993)$ et Le pays maudit* (1995) qui s'attachent à dénoncer une réalité sournoise et cauchemardesque, celle des « années noires » de la dernière décennie du $\mathrm{XX}^{\mathrm{e}}$ siècle. Le monde de ces romans, peuplé de personnages ubuesques, est présenté sous forme d'une farce tragi-comique où le grotesque côtoie l'absurde, une farce qui suscite chez le lecteur un rire sardonique.

Si l'actualité dramatique et les événements bouleversants des années 1990 captivent l'attention de la plupart des écrivains, ils ne sont pas, naturellement, de sujets uniques dans la littérature serbe de cette époque. Attiré plutôt par les questions d'ordre métaphysique et ontologique ou par celles relevant de l'esthétique, un certain nombre d'écrivains restent fidèles à leurs thèmes de prédilection qui ne sont pas en prise directe avec l'actualité. À titre d'exemple citons Petković, un érudit à l'esprit d'explorateur et à l'imagination débordante. Romancier et nouvelliste, cet écrivain a publié, durant les années 1990, seulement deux livres, mais tous les deux fortement appréciés par la critique : le roman Destin et commentaires* (1993) et le recueil de novelles L'homme qui vivait dans les rêves* (1998). À la fois roman à énigme et vaste fresque pseudo-historique sur la diaspora serbe et ses fantasmes, Destin et commentaires est également, d'une certaine 
manière, un roman sur le roman vu commeun laboratoire à fabriquer des histoires. Quant aux nouvelles de Petković, elles sont aussi les produits d'un jeu subtil de l'érudition et de l'imagination. Conçues sur les procédés borgésiens ou sur ceux relevant des stratégies narratives postmodernes, elles se réfèrent sans cesse à l'histoire, à la religion et à la mythologie, et introduisent le lecteur dans un univers insolite. Les plus originales mais aussi les plus déroutantes parmi ces nouvelles sont celles qui relèvent du réalisme magique, celles qui laissent présager l'étrange coexistence de mondes parallèles: le visible et l'invisible, le réel et le fantastique.

Pour compléter ce panorama rapide de la littérature serbe de la dernière décennie du $\mathrm{XX}^{\mathrm{e}}$ siècle, il est indispensable de dire quelques mots encore sur Pavić, le pape du postmodernisme serbe disparu récemment. Cet écrivain de renom international qui s'est lancé dès le départ dans un projet littéraire extraordinaire dont le but était non seulement de renouveler l'art de la narration mais aussi de changer le mode traditionnel de lecture, n'a cessé, durant les années 1990, de surprendre son public en se jetant dans des nouvelles investigations déroutantes. Et si son esprit ludique semble atteindre son sommet dans le Dictionnaire khazar, Pavić a démontré dans ses nouveaux livres qu'il possède toujours une énergie créatrice débordante, la preuve que ses sources novatrices ne sont pas encore taries. En effet, ses romans publiés ultérieurement représentent, chacun à leur tour, de nouvelles réalisations de l'idée de "l'œuvre ouverte » appliquée déjà dans son fameux roman-lexique, l'idée qui compte sur la complicité et l'imagination du lecteur. Ainsi dans L'Envers du vent* (1991), l'auteur réalise un nouveau modèle romanesque : une sorte de roman-sablier. Concrètement, cette œuvre, composée de deux parties disposées tête-bêche, possède deux débuts et une seule fin, située au milieu du livre : une fois lue la moitié de l'ouvrage, nous devons ensuite le prendre à l'envers pour en continuer la lecture comme on retourne un sablier. Enfin, citons Le dernier amour à Constantinople*(1994),roman conçu selon les règles du tarot, destiné avant tout aux amateurs de jeux de cartes et de cartomancie. Au-delà de sa forme insolite, ce dernier livre montre une fois de plus, que son auteur reste toujours l'insaisissable « maitre de la voltige » qui prend plaisir à dépasser les limites du roman et de la littérature en général.

\section{Conclusion}

Ce court aperçu de l'évolution de la littérature serbe durant les deux décennies charnières, celle qui a précédé la chute du Mur de Berlin et celle qui lui a succédé - aurait pu évidemment être bien plus large. Mais les auteurs et les livres évoqués peuvent déjà donner, nous semble-t-il, une idée assez précise sur la production littéraire en Serbie durant cette époque agitée, en particulier sur l'état d'esprit des écrivains, sur leurs orientations stylistique et thématique ou encore sur leur choix idéologique et éthique. D'autre part, cet aperçu avait pour objet également d'éclaircir et de dessiner le contexte socio-historique particulièrement complexe dans lequel a évolué la littérature serbe contrainte de naviguer en permanence entre deux dangers : le marteau de l'idéologie et l'enclume de l'Histoire. Ainsi on a pu se rendre compte de quelle manière cette littérature a réussi - malgré plusieurs chocs de la grande Histoire et les pressions exercées par les régimes autoritaires, d'abord celui de Tito et de ses successeurs et puis celui de Milošević - à surmonter toutes les épreuves et sauvegarder son autonomie, du moins quand il s'agit de ses meilleurs écrivains. C'est une preuve, une de plus, que les grands bouleversements historiques et la crise de la société n'entravent pas forcément les facultés créatrices et 
artistiques. Heureusement, ce contexte socio-historique ainsi que les défis à relever qui en découlent changeront considérablement après la chute de Milošević et l'instauration de la démocratie en Serbie en l'an 2000. Certes, les écrivains, enfin libres de respirer à pleins poumons, reviendront encore souvent aux thèmes liés à l'époque des années 1990, époque dramatique qui a laissé, dans la conscience collective, des traumatismes profonds et des blessures à peine cicatrisées. Mais, dans leurs livres, s'imposent de plus en plus des thèmes plus universels et un ton plus intimiste : signes d'un nouvel état d'esprit et d'un nouveau tournant dans la littérature serbe.

\section{BIBLIOGRAPHIE}

Albahari David, Snežni čovek, Vreme knjige, Belgrade, 1995 / L'homme de neige, traduit du serbe par Gojko Lukić et Gabriel Iaculli, Gallimard, Paris, 2003.

Albahari David, Mamac, Stubovi kulture, Belgrade, 1996 / L'appât, traduit du serbe par Gojko Lukić et Gabriel Iaculli, Gallimard, Paris, 1999.

Albahari David, Mrak, Narodna knjiga-Alfa, Belgrade 1997.

Albahari David, Gec i Majer, Stubovi kulture, Belgrade, 1998 / Goetz et Meyer, traduit du serbe par Gabriel Iaculli et Gojko Lukić, Gallimard, Paris, 2002

Basara Svetislav, De bello civili, Vreme Publikum, Belgrade, 1993 / De bello civili : version Vitamine C, traduit du serbo-croate par Alain Cappon, Gaïa éditions, Larbey, 1996.

Basara Svetislav, Ukleta zemlja, Vreme knjige, Belgrade, 1995 / Le pays maudit, traduit du serbocroate par Alain Cappon, Gaïa éditions, Larbey 1998.

Ćosić Dobrica, Vreme zla, Prosveta, BIGZ, Belgrade, 1996 / Le temps du mal ; Le pécheur ; L'hérétique, traduit du serbo-croate par Slobodan Despot, L'Age d'homme, Lausanne, 1990.

Danojlić Milovan, Pisati pod nadzorom, Nova Jugoslavia, Belgrade, 1987 / Écrire sous surveillance, traduit du serbe par Vesna Bernard-Radović, Poésie-bis, Paris, 1987.

Djogo Gojko, Vunena vremena, Prosveta, Belgrade, 1981.

Drašković Vuk, Nož, Zapis, Belgrade, 1982 / Le Couteau, traduit du serbe par Irina Danil, édition revue par Elie Robert Nicoud, J.-C. Lattès, Paris, 1993.

Đuretić Veselin, Saveznici i jugoslovenska ratna drama, Multiprint, Belgrade, 1986.

Koštunica Vojislav \& Čavoški Kosta, Stranački pluralizam ili monizam :društveni pokreti i politički sistem u Jugoslaviji 1944-1949, Centar za filozofiju i društvenu teoriju, Belgrade, 1983.

Kovačević Dušan, Balkanski špijun, BIGZ, Belgrade, 1983.

Isaković Antonije, Tren, Prosveta, Belgrade, 1982.

Mihajlović Dragoslav, Kad su cvetale tikve, Matica Srpska, Novi Sad, 1968 / Quand les courges étaient en fleurs, traduit du serbo-croate par Jean Descat, Gallimard, Paris, 1972. 
Mihailović Dragoslav, Goli otok. Knj, Politika, Belgrade, 1990 ; Goli otok knj. 1 \& 2, SKZ, Belgrade, 1995.

Pavić Milorad, Hazarski rečnik : roman leksikon u 100000 reči, Prosveta, BIGZ, Belgrade, 1984 / Le Dictionnaire Khazar : roman-lexique en 100000 mots, traduit du serbo-croate par Maria Bežanovska, Belfond, Paris, 1988.

Pavić Milorad, Poslednja ljubav u Carigradu : priručnik za gatanje, Prosveta, Belgrade, 1994 / Dernier amour à Constantinople, traduit du serbo-croate par Jean Descat, Noir sur Blanc, Montricher, 2000.

Pavić Milorad, Unutrašnja strana vetra ili Roman o Heri i Leandru, Prosveta, Belgrade, 1991 / L'Envers du vent, traduit du serbe par Madeline Stevanov, Belfond, Paris, 1992

Petković Radoslav, Čovek koji je živeo u snovima, Stubovi kulture, Belgrade, 1998 / L'homme qui vivait dans les rêves, traduit du serbo-croate par Alain Cappon, Gaïa éditions, Larbey, 1999.

Petković Radoslav, Sudbina i komentari, Vreme, Belgrade, 1993 / Destin et commentaire, traduit du serbo-croate par Alain Cappon, Gaïa éditions, Larbey, 1998.

Popović Danko, Knjiga o Milutinu, Književne novine, Belgrade,1984 / Le Livre de Miloutine, traduit du serbo-croate par Jean Descat, Stock, Paris, 1989.

Popović Miroslav, Udri bandu, Filip Višnjić, Belgrade, 1988 / Les vauriens de Tito, traduit du serbo-croate par Pascale Delpech, Christian Bourgois, Paris, 1991.

Radulović Jovan, Golubnjača, Srpska književna zadruga, Belgrade, 1980.

Radulović Jovan, Idealan plac, Filip Višnjić, Belgrade, 2000.

Radulović Jovan, Prošao život, Srpska književna zadruga, Belgrade, 1997.

Savić Milisav, Hleb i strah, Srpska književna zadruga,Belgrade, 1991.

Selenić Slobodan, Pismo-glava, Prosveta, Belgrade, 1982.

Stevanović Vidosav, Abel i Liza, LIR BG, Belgrade, 2001 / Abel et Lise, traduit du serbe par Nicole Dizdarević \& Mauricette Begic, L’Esprit des péninsules, coll. « Balkaniques », Paris, 2003.

Stevanović Vidosav, Hristos i psi, Radio B 92, Belgrade, 1994 / Christ et les chiens, traduit du serbo-croate par Mauricette Begić, Belfond, Paris, 1993.

Stevanović Vidosav, Ista stvar, Oktoih, Belgrade, 2004 / La même chose, traduit du serbe par Mauricette Begic \& Nicole Dizdarević, Mercure de France, Paris, 1999.

Stevanović Vidosav, Sneg u Atini, Nova Svetlost, Kragujevac, 1992 / La neige et les chiens, traduit du serbo-croate par Mauricette Begić \& Christine Chaton, Belfond, Paris, 1993.

Velmar-Janković Svetlana, Lagum, Belgrade, BIGZ, 1990 / Dans le noir, traduit du serbo-croate par Alain Cappon, Phébus, Paris 1997.

Velmar-Janković Svetlana, Nigdina, Stubovi kulture, Belgrade, 2000 / Le pays de nulle part, traduit du serbo-croate par Alain Cappon, Phébus, Paris, 2001.

Velikić Dragan, Severni zid, Vreme knjige, Belgrade, 1995 / Le Mur nord, traduit du serbo-croate par Alain Cappon, Gaïa éditions, Larbey, 2001 


\section{NOTES}

1. Les titres de tous les ouvrages mentionnés dans ce texte sont, pour faciliter leur lecture et leur compréhension, donnés en version française. Pour distinguer ceux d'entre eux qui sont traduits en français, leurs titres sont suivi d'un astérisque $\left(^{*}\right)$. Le lecteur trouvera les informations complètes aussi bien sur la version originale que sur la version traduite de toutes les œuvres évoquées dans la bibliographie annexée au présent article.

2. Comme l'a justement prévu Bernard Chapuis, « il y a fort à parier que, si la chose est possible, on gardera au pouvoir le fantôme de ce grand absent qui ne voulait pas devenir un grand disparu » (« Un grand absent », in « LE MONDE », 6 mai 1980, p. 1).

3. Signalons toutefois que Dragoslav Mihailović fut le premier à évoquer l'année 1948, ce qui lui avait, bien entendu, valu d'être sanctionné. Plus précisément, la rupture fracassante entre Tito et Staline ainsi que le "goulag yougoslave" sont évoqués dans son livre-maître intitulé Quand les courges étaient en fleurs* (1968), certes en filigrane, mais sans avoir pu déjouer pour autant la vigilance de la censure qui interdira la pièce de théâtre adaptée de ce roman. Faut-il ajouter que les censeurs savaient très bien que ce livre "hérétique" faisait également allusion à une expérience traumatisante de l'écrivain ? En effet, en 1950, Mihailović fut arrêté et incarcéré dans le camp de Goli otok où il aura l'occasion de faire la terrible expérience des méthodes de « rééducation » du régime titiste. Parmi d'autres livres écrits sur ce sujet notons en particulier celui de Miroslav Popović, victime lui aussi des purges titistes (voir son livre Udri Bandu*, 1988).

4. La partie n'était cependant pas encore gagnée. Le régime a continué, certes avec plus de prudence, à sanctionner les livres qu'il trouvait les plus compromettants pour ses intérêts. En 1983 il a, par exemple, interdit l'ouvrage de Kosta Čavoški et Vojislav Koštunica : Le pluralisme de partis ou le monisme. La même mesure a été appliquée contre l'étude de Veselin Đuretić, les Alliés et le drame de guerre yougoslave, en 1987.

5. Même s'il s'agit avant tout d'un ouvrage ludique explorant de nouvelles formes de narration, ouvrage qui de surcroît évoque l'histoire d'un peuple disparu, cela n'a pas empêché certains critiques de voir en lui un livre engagé et même " une parabole du destin serbe ». Citons, à ce propos, deux articles rédigés par les critiques américains. Le premier est publié dans le mensuel de Washington «THE WORLD » le $1^{\mathrm{er}}$ novembre 1988. «Dans le livre vert, en décrivant les Khazars, constate le critique américain, Pavić se rapproche tout à fait d'une identification de la position actuelle des Serbes en Yougoslavie avec celle de Khazars." En développant la même comparaison, la seconde revue «PHILADELPHIA INQUIRER »(décembre 1988) va encore plus loin : « De même que les Serbes dépités (sic!) par leur position en Yougoslavie, les Khazars subissent une discrimination tout en étant majoritaires dans leur pays. Les minorités qui, avec un soutien extérieur, gagnent en force politique dans certaines parties de l'État Khazar, réduisent la population khazar locale, de même que la minorité albanaise ainsi que les autres groupes ethniques exercent une discrimination à l'égard des Serbes habitant ces régions. C'est cette injustice, servant à Pavić d'allusion claire pour ses lecteurs yougoslaves, qui mène à la disparition des Khazars... »

6. Selon Slobodan Rakitić, un certain nombre de poèmes des auteurs mentionnés appartiennent à un genre poétique particulier qui, durant les années 1980, devient de plus en plus important. Il s'agit de la " poésie politique » qui observe l'actualité à travers un prisme critique sans utiliser forcément le langage métaphorique. Pour autant, chez les meilleurs poètes, elle ne se limite pas, précise-t-il, à une simple interprétation de la réalité au premier degré. (Rakitić $\mathrm{S}$. : «Politička poezija " (La Poésie politique), in:Novija srpska književnost $i$ kritika ideologije (La nouvelle littérature serbe et la critique de l'idéologie), SANU, knj. XLVI, Belgrade-Niš, 1989, pp. 107-113). 
7. Les termes " postmoderne » et "postmodernisme », en Serbie comme ailleurs, restent bien sûr assez flous et imprécis. Ils sont venus d'outre-Atlantique, avec les traductions des auteurs américains contemporains qui les revendiquaient et qui ont eu un certain impact sur la jeune génération des écrivains serbes apparus sur la scène littéraire pendant les années 1980 ou dès la fin des années 1970.

8. Cette image de la littérature serbe des années 1980, où dominent trois courants littéraires distincts, est une image quelque peu schématisée et simplifiée pour les besoins de notre analyse. Il ne s'agit pas, rappelons-le encore, de courants monolithiques et homogènes qui seraient nettement séparés ou opposés. En fait, les deux premiers se rejoignent dans ce que Predrag Palavestra appelle «la littérature critique » qui met en cause l'idéologie autant que la réalité, mais qui englobe, précise Palavestra, des œuvres très différentes par l'écriture (Palavestra P.: « Novija srpska književnost i kritika ideologije » (La Nouvelle Littérature serbe et la critique de l'idéologie), in : Novija srpska književnost $i$ kritika ideologije, Op. cit., pp. 1-8.). Signalons à ce propos un autre terme proposé par Igor Mandić : «le roman de la crise». Ce terme englobe, selon ce critique, la majorité des œuvres romanesques, publiées en Serbie et en Croatie durant «l'époque post-titiste ", y compris celles de certains auteurs appartenant aux trois courants que nous avons cités. (Mandić I. : « Romani krize » (Les Romans de la crise), in : Ibid., pp. 65-67).

9. En quittant leur pays, ces écrivains ainsi qu'un certain nombre de leurs confrères qui ont aussi choisi le chemin de l'exil durant les années 1990, n'ont pas quitté leur langue maternelle. Ils ont continué d'écrire et de publier en serbe.

\section{INDEX}

Mots-clés : littérature serbe

Index géographique : Serbie

Index chronologique : communisme, XXe siècle, post-communisme

\section{AUTEURS}

MILIVOJ SREBRO

Maître de Conférences à l'Université Bordeaux III 